\title{
Design Serial Intelligent Modified Feedback Linearization like Controller with Application to Spherical Motor
}

\author{
Ali Barzegar, Farzin Piltan, Mahmood Vosoogh, Abdol Majid Mirshekaran, Alireza Siahbazi \\ Research and Development Department, Institute of Advance Science and Technology-IRAN SSP, Shiraz, Iran \\ E-mail: SSP.ROBOTIC@IRANSSP.COM; http://WWW.IRANSSP.COM
}

\begin{abstract}
Fuzzy logic controller (FLC) is an important nonlinear controller in an uncertain dynamic system's parameters. This controller is used to control of nonlinear dynamic systems particularly for spherical motor, because it has a suitable control performance and it is a stable. Conversely pure fuzzy logic controller is a high-quality intelligent nonlinear controller; it has two important problems; reliability and robustness in uncertain dynamic parameter. To increase the reliability and robustness, this research is focused on applied feedback linearization method in pure fuzzy logic controller. In this research the nonlinear equivalent dynamic (equivalent part) formulation problem in uncertain condition is also solved by combine pure fuzzy logic control and feedback linearization method. In this method feedback linearization theorem is applied to fuzzy logic controller to increase the stability, reliability and robustness, which it is based on nonlinear dynamic formulation. To achieve this goal, the dynamic-based formulation feedback linearization method is design. This method is robust and model-based nonlinear control therefore can reduce the nonlinearity term of system and reduce the effect of coupling. In this research MAMDANI fuzzy inference system is used as a main controller. It has minimum rule base to practical implementation. This technique was employed to obtain the desired control behavior with a number of information about dynamic model of system and a feedback linearization control was applied to reinforce system performance.
\end{abstract}

Index Terms - Fuzzy Logic Theory, Feedback Linearization Method, Spherical Motor, Feedback Linearization like Controller

\section{Introduction and Background}

Multi-degree-of-freedom (DOF) actuators are finding wide use in a number of Industries. Currently, a significant number of the existing robotic actuators that can realize multi-DOF motion are constructed using gear and linkages to connect several single-DOF motors in series and/or parallel. Not only do such actuators tend to be large in size and mass, but they also have a decreased positioning accuracy due to mechanical deformation, friction and backlash of the gears and linkages. A number of these systems also exhibit singularities in their workspaces, which makes it virtually impossible to obtain uniform, high-speed, and high-precision motion. For high precession trajectory planning and control, it is necessary to replace the actuator system made up of several single-DOF motors connected in series and/or parallel with a single multi-DOF actuator. The need for such systems has motivated years of research in the development of unusual, yet high performance actuators that have the potential to realize multi-DOF motion in a single joint. One such actuator is the spherical motor. Compared to conventional robotic manipulators that offer the same motion capabilities, the spherical motor possesses several advantages. Not only can the motor combine 3-DOF motion in a single joint, it has a large range of motion with no singularities in its workspace. The spherical motor is much simpler and more compact in design than most multiple single-axis robotic manipulators. The motor is also relatively easy to manufacture. The spherical motor have potential contributions to a wide range of applications such as coordinate measuring, object tracking, material handling, automated assembling, welding, and laser cutting. All these applications require high precision motion and fast dynamic response, which the spherical motor is capable of delivering. Previous research efforts on the spherical motor have demonstrated most of these features. These, however, come with a number of challenges. The spherical motor exhibits coupled, nonlinear and very complex dynamics. The design and implementation of feedback controllers for the motor are complicated by these dynamics. The controller design is further complicated by the orientation-varying torque generated by the spherical motor. Some of these challenges have been the focus of previous and ongoing research [1-11].

In modern usage, the word of control has many meanings, this word is usually taken to mean regulate, direct or command. The word feedback plays a vital role in the advance engineering and science. The conceptual frame work in Feed-back theory has developed only since world war II. In the twentieth century, there was a rapid growth in the application of feedback controllers in process industries. According to Ogata, to do the first 
significant work in three-term or PID controllers which Nicholas Minorsky worked on it by automatic controllers in 1922. In 1934, Stefen Black was invention of the feedback amplifiers to develop the negative feedback amplifier[12-28]. Negative feedback invited communications engineer Harold Black in 1928 and it occurs when the output is subtracted from the input. Automatic control has played an important role in advance science and engineering and its extreme importance in many industrial applications, i.e., aerospace, mechanical engineering and joint control. The first significant work in automatic control was James Watt's centrifugal governor for the speed control in motor engine in eighteenth century[29-40]. There are several methods for controlling a spherical motor, which all of them follow two common goals, namely, hardware/software implementation and acceptable performance. However, the mechanical design of spherical motor is very important to select the best controller but in general two types schemes can be presented, namely, a joint space control schemes and an operation space control schemes[41-57]. Joint space and operational space control are closed loop controllers which they have been used to provide robustness and rejection of disturbance effect. The main target in joint space controller is to design a feedback controller which the actual motion $\left(\boldsymbol{q}_{\boldsymbol{a}}(\boldsymbol{t})\right)$ and desired motion $\left(\boldsymbol{q}_{\boldsymbol{d}}(\boldsymbol{t})\right)$ as closely as possible. This control problem is classified into two main groups. Firstly, transformation the desired motion $\boldsymbol{X}_{\boldsymbol{d}}(\boldsymbol{t})$ to joint variable $\boldsymbol{q}_{\boldsymbol{d}}(\boldsymbol{t})$ by inverse kinematics of spherical motor[34-50]. This control includes simple PD control, PID control, inverse dynamic control, Lyapunov-based control, and passivity based control. The main target in operational space controller is to design a feedback controller to allow the actual end-effector motion $\boldsymbol{X}_{\boldsymbol{a}}(\boldsymbol{t})$ to track the desired endeffector motion $\boldsymbol{X}_{\boldsymbol{d}}(\boldsymbol{t})$. This control methodology requires a greater algorithmic complexity and the inverse kinematics used in the feedback control loop. Direct measurement of operational space variables are very expensive that caused to limitation used of this controller in spherical motor[53-55]. One of the simplest ways to analysis control of three DOF spherical motor are analyzed each joint separately such as SISO systems and design an independent joint controller for each joint. In this controller, inputs only depends on the velocity and displacement of the corresponding joint and the other parameters between joints such as coupling presented by disturbance input. Joint space controller has many advantages such as one type controllers design for all joints with the same formulation, low cost hardware, and simple structure. A nonlinear methodology is used for nonlinear uncertain systems (e.g., spherical motor) to have an acceptable performance. These controllers divided into six groups, namely, feedback linearization (computed-torque control), passivity-based control, sliding mode control (variable structure control), artificial intelligence control, lyapunov-based control and adaptive control[13-26].
In recent years, artificial intelligence theory has been used to control of highly nonlinear systems. Neural network, fuzzy logic and neuro-fuzzy are synergically combined with nonlinear classical controller and used in nonlinear, time variant and uncertain plant (e.g., spherical motor). Fuzzy logic controller (FLC) is one of the most important applications of fuzzy logic theory. This controller can be used to control nonlinear, uncertain, and noisy systems. This method is free of some model techniques as in model-based controllers. As mentioned that fuzzy logic application is not only limited to the modelling of nonlinear systems [31-36] but also this method can help engineers to design a model-free controller. Control spherical motor using model-based controllers are based on manipulator dynamic model. These controllers often have many problems for modelling. Conventional controllers require accurate information of dynamic model of spherical motor, but most of time these models are MIMO, nonlinear and partly uncertain therefore calculate accurate dynamic model is complicated [32]. The main reasons to use fuzzy logic methodology are able to give approximate recommended solution for uncertain and also certain complicated systems to easy understanding and flexible. Fuzzy logic provides a method to design a model-free controller for nonlinear plant with a set of IF-THEN rules [32]. This paper contributes to the research effort of alternate methods for modelling the torque generated by the spherical motor used in the feedback linearization like controller design. The designed controller not only demonstrates the appealing features exhibited by the spherical motor, but also demonstrates some of the nice features of fuzzy controllers as well.

Feedback linearization controller (FLC) is a powerful nonlinear controller which has been analyzed by many researchers especially in recent years. The main reason to opt for this controller is its acceptable control performance in wide range and solves two most important challenging topics in control which names, stability and robustness [24-57]. Feedback linearization controller is divided into two main sub controllers: linear part controller $\left(\boldsymbol{\tau}_{\text {lin }}\right)$ and equivalent controller $\left(\boldsymbol{\tau}_{\text {eq }}\right)$. Linear part controller causes an acceptable tracking performance at the expense of the coefficient of proportional, derivative and integral part. After going toward the reference trajectory by linear term, equivalent term help to the system dynamics match to the reference trajectory [12-15]. However, this technique used in many applications but, pure computed torque controller has following challenges: robustness in presence of disturbance, and nonlinear equivalent dynamic formulation [20].This controller works very well when all dynamic and physical parameters are known but when the system has variation in dynamic parameters, the controller has no acceptable performance[14]. In practice, most of physical systems (e.g., spherical motor) parameters are unknown or time variant, therefore, feedback linearization like controller used to compensate dynamic equation of spherical motor [15-23]. 
This paper is organized as follows; second part focuses on the modelling dynamic formulation based on Lagrange methodology, fuzzy logic controller and feedback linearization controller to estimates the challenge of fuzzy logic theory. Third part is focused on the methodology which can be used to reduce the error, increase the performance quality and increase the robustness and stability. Simulation result and discussion is illustrated in forth part which based on trajectory following and disturbance rejection. The last part focuses on the conclusion and compare between this method and the other ones.

\section{Theory}

\subsection{Dynamic and Kinematics Formulation of Spherical Motor}

Dynamic modeling of spherical motors is used to describe the behavior of spherical motor such as linear or nonlinear dynamic behavior, design of model based controller such as pure sliding mode controller which design this controller is based on nonlinear dynamic equations, and for simulation. The dynamic modeling describes the relationship between motion, velocity, and accelerations to force/torque or current/voltage and also it can be used to describe the particular dynamic effects (e.g., inertia, coriolios, centrifugal, and the other parameters) to behavior of system[1-10]. Spherical motor has a nonlinear and uncertain dynamic parameters 3 degrees of freedom (DOF) motor.

The equation of a spherical motor governed by the following equation [1-10]:

$$
H(q)\left[\begin{array}{c}
\ddot{\alpha} \\
\ddot{\beta} \\
\ddot{\gamma}
\end{array}\right]+B(q)\left[\begin{array}{c}
\dot{\alpha} \dot{\beta} \\
\dot{\alpha} \dot{\gamma} \\
\dot{\beta} \dot{\gamma}
\end{array}\right]+C(q)\left[\begin{array}{c}
\dot{\alpha}^{2} \\
\dot{\beta}^{2} \\
\dot{\gamma}^{2}
\end{array}\right]=\left[\begin{array}{c}
\tau_{x} \\
\tau_{y} \\
\tau_{z}
\end{array}\right]
$$

Where $\tau$ is actuation torque, $\mathrm{H}(\mathrm{q})$ is a symmetric and positive define inertia matrix, $\mathrm{B}(\mathrm{q})$ is the matrix of coriolios torques, $\mathrm{C}(\mathrm{q})$ is the matrix of centrifugal torques.

This is a decoupled system with simple second order linear differential dynamics. In other words, the component $\ddot{q}$ influences, with a double integrator relationship, only the variable $q_{i}$, independently of the motion of the other parts. Therefore, the angular acceleration is found as to be [1-11]:

$$
\ddot{\boldsymbol{q}}=\boldsymbol{H}^{-1}(\boldsymbol{q}) \cdot\{\boldsymbol{\tau}-\{\boldsymbol{B}+\boldsymbol{C}\}\}
$$

This technique is very attractive from a control point of view.

Study of spherical motor is classified into two main groups: kinematics and dynamics. Calculate the relationship between rigid bodies and final part without any forces is called Kinematics. Study of this part is pivotal to design with an acceptable performance controller, and in real situations and practical applications. As expected the study of kinematics is divided into two main parts: forward and inverse kinematics. Forward kinematics has been used to find the position and orientation of task frame when angles of joints are known. Inverse kinematics has been used to find possible joints variable (angles) when all position and orientation of task frame be active [1].

The main target in forward kinematics is calculating the following function:

$$
\boldsymbol{\Psi}(\boldsymbol{X}, \boldsymbol{q})=\mathbf{0}
$$

Where $\Psi(.) \in R^{n}$ is a nonlinear vector function, $X=\left[X_{1}, X_{2}, \ldots \ldots, X_{l}\right]^{T}$ is the vector of task space variables which generally task frame has three task space variables, three orientation, $q=\left[q_{1}, q_{2}, \ldots, q_{n}\right]^{T}$ is a vector of angles or displacement, and finally $n$ is the number of actuated joints. The Denavit-Hartenberg (D-H) convention is a method of drawing spherical motor free body diagrams. Denvit-Hartenberg (D-H) convention study is necessary to calculate forward kinematics in this motor.

A systematic Forward Kinematics solution is the main target of this part. The first step to compute Forward Kinematics (F.K) is finding the standard D-H parameters. The following steps show the systematic derivation of the standard D-H parameters.

1. Locate the spherical motor

2. Label joints

3. Determine joint rotation $(\theta)$

4. Setup base coordinate frames.

5. Setup joints coordinate frames.

6. Determine $\alpha_{i}$, that $\alpha_{i}$, link twist, is the angle between $Z_{i}$ and $Z_{i+1}$ about an $X_{i}$.

7. Determine $d_{i}$ and $a_{i}$, that $a_{i}$, link length, is the distance between $Z_{i}$ and $Z_{i+1}$ along $X_{i} . d_{i}$, offset, is the distance between $X_{i-1}$ and $X_{i}$ along $Z_{i}$ axis.

8. Fill up the D-H parameters table. The second step to compute Forward kinematics is finding the rotation matrix $\left(R_{n}^{0}\right)$. The rotation matrix from $\left\{F_{i}\right\}$ to $\left\{F_{i-1}\right\}$ is given by the following equation;

$$
R_{i}^{i-1}=U_{i\left(\theta_{i}\right)} V_{i\left(\alpha_{i}\right)}
$$

Where $U_{i\left(\theta_{i}\right)}$ is given by the following equation [1-11];

$$
U_{i\left(\theta_{i}\right)}=\left[\begin{array}{ccc}
\cos \left(\theta_{i}\right) & -\sin \left(\theta_{i}\right) & 0 \\
\sin \left(\theta_{i}\right) & \cos \left(\theta_{i}\right) & 0 \\
0 & 0 & 1
\end{array}\right]
$$

and $V_{i\left(\alpha_{i}\right)}$ is given by the following equation [1-11];

$$
V_{i\left(\theta_{i}\right)}=\left[\begin{array}{ccc}
1 & 0 & 0 \\
0 & \cos \left(\alpha_{i}\right) & -\sin \left(\alpha_{i}\right) \\
0 & \sin \left(\alpha_{i}\right) & \cos \left(\alpha_{i}\right)
\end{array}\right]
$$


So $\left(R_{n}^{0}\right)$ is given by [8]

$$
R_{n}^{\mathbf{0}}=\left(U_{1} V_{1}\right)\left(U_{2} V_{2}\right) \ldots \ldots\left(U_{n} V_{n}\right)
$$

The final step to compute the forward kinematics is calculate the transformation ${ }_{n}^{0} T$ by the following formulation [3]

$$
{ }_{n}^{0} T={ }_{1}^{0} T \cdot{ }_{2}^{1} T \cdot{ }_{3}^{2} T \ldots \ldots \cdot{ }_{n}^{n-1} T=\left[\begin{array}{cc}
R_{n}^{0} & 0 \\
0 & 1
\end{array}\right]
$$

\subsection{Fuzzy Logic Theory}

This section provides a review about foundation of fuzzy logic based on [32- 53]. Supposed that $U$ is the universe of discourse and $x$ is the element of $U$, therefore, a crisp set can be defined as a set which consists of different elements $(x)$ will all or no membership in a set. A fuzzy set is a set that each element has a membership grade, therefore it can be written by the following definition;

$$
\boldsymbol{A}=\left\{\boldsymbol{x}, \boldsymbol{\mu}_{\boldsymbol{A}}(\boldsymbol{x}) \mid \boldsymbol{x} \in \boldsymbol{X}\right\} ; \boldsymbol{A} \in \boldsymbol{U}
$$

Where an element of universe of discourse is $x, \mu_{A}$ is the membership function (MF) of fuzzy set. The membership function $\left(\mu_{A}(x)\right)$ of fuzzy set $A$ must have a value between zero and one. If the membership function $\mu_{A}(x)$ value equal to zero or one, this set change to a crisp set but if it has a value between zero and one, it is a fuzzy set. Defining membership function for fuzzy sets has divided into two main groups; namely; numerical and functional method, which in numerical method each number has different degrees of membership function and functional method used standard functions in fuzzy sets. The membership function which is often used in practical applications includes triangular form, trapezoidal form, bell-shaped form, and Gaussian form.

Linguistic variable can open a wide area to use of fuzzy logic theory in many applications (e.g., control and system identification). In a natural artificial language all numbers replaced by words or sentences.

If - then Rule statements are used to formulate the condition statements in fuzzy logic. A single fuzzy If then rule can be written by

\section{If $x$ is $A$ Then $y$ is $B$}

where $A$ and $B$ are the Linguistic values that can be defined by fuzzy set, the If - part of the part of " $x$ is $A$ " is called the antecedent part and the then part of the part of " $y$ is $B$ " is called the Consequent or Conclusion part. The antecedent of a fuzzy if-then rule can have multiple parts, which the following rules shows the multiple antecedent rules:

\section{if $e$ is $N B$ and $\dot{e}$ is $M L$ then $T$ is $L L$}

where $e$ is error, $\dot{e}$ is change of error, $N B$ is Negative Big, $M L$ is Medium Left, $T$ is torque and $L L$ is Large Left. If - then rules have three parts, namely, fuzzify inputs, apply fuzzy operator and apply implication method which in fuzzify inputs the fuzzy statements in the antecedent replaced by the degree of membership, apply fuzzy operator used when the antecedent has multiple parts and replaced by single number between 0 to 1 , this part is a degree of support for the fuzzy rule, and apply implication method used in consequent of fuzzy rule to replaced by the degree of membership. The fuzzy inference engine offers a mechanism for transferring the rule base in fuzzy set which it is divided into two most important methods, namely, Mamdani method and Sugeno method. Mamdani method is one of the common fuzzy inference systems and he designed one of the first fuzzy controllers to control of system engine. Mamdani's fuzzy inference system is divided into four major steps: fuzzification, rule evaluation, aggregation of the rule outputs and defuzzification. Michio Sugeno use a singleton as a membership function of the rule consequent part. The following definition shows the Mamdani and Sugeno fuzzy rule base

$$
\begin{array}{rr}
\text { Mamdani } F . R^{1}: \text { if } & x \text { is } A \text { and } \\
y \text { is } B \text { then } & \text { z is } C \\
\text { Sugeno } F . R^{1}: \text { if } & x \text { is } A \text { and } \\
y \text { is } B \text { then } & f(x, y) \text { is } C
\end{array}
$$

When $x$ and $y$ have crisp values fuzzification calculates the membership degrees for antecedent part. Rule evaluation focuses on fuzzy operation $(A N D / O R)$ in the antecedent of the fuzzy rules. The aggregation is used to calculate the output fuzzy set and several methodologies can be used in fuzzy logic controller aggregation, namely, Max-Min aggregation, Sum-Min aggregation, Max-bounded product, Max-drastic product, Max-bounded sum, Max-algebraic sum and Min-max. Two most common methods that used in fuzzy logic controllers are Max-min aggregation and Sum-min aggregation. Max-min aggregation defined as below

$$
\begin{aligned}
& \mu_{U}\left(x_{k}, y_{k}, U\right)=\mu_{\cup_{i=1}^{r} F R^{i}}\left(x_{k}, y_{k}, U\right) \\
& =\max \left\{\min _{i=1}^{r}\left[\mu_{R_{p q}}\left(x_{k}, y_{k}\right), \mu_{p_{m}}(U)\right]\right\}
\end{aligned}
$$

The Sum-min aggregation defined as below

$$
\begin{aligned}
& \mu_{U}\left(x_{k}, y_{k}, U\right)=\mu_{\cup_{i=1}^{r} F R^{i}}\left(x_{k}, y_{k}, U\right) \\
& =\sum \min _{i=1}^{r}\left[\mu_{R q q}\left(x_{k}, y_{k}\right), \mu_{p_{m}}(U)\right]
\end{aligned}
$$

where $r$ is the number of fuzzy rules activated by $x_{k}$ and $y_{k}$ and also $\mu_{\cup_{i=1}^{r} F R}\left(x_{k}, y_{k}, U\right)$ is a fuzzy interpretation of $i-t h$ rule. Defuzzification is the last step in the fuzzy inference system which it is used to 
transform fuzzy set to crisp set. Consequently defuzzification's input is the aggregate output and the defuzzification's output is a crisp number. Centre of gravity method (COG) and Centre of area method $(C O A)$ are two most common defuzzification methods, which COG method used the following equation to calculate the defuzzification

$$
\operatorname{COG}\left(x_{k}, y_{k}\right)=\frac{\sum_{i} U_{i} \sum_{j=1}^{r} \cdot \mu_{u}\left(x_{k}, y_{k}, U_{i}\right)}{\sum_{i} \sum_{j=1}^{r} \cdot \mu_{u}\left(x_{k}, y_{k}, U_{i}\right)}
$$

and COA method used the following equation to calculate the defuzzification

$$
\operatorname{COA}\left(x_{k}, y_{k}\right)=\frac{\sum_{i} U_{i} \cdot \mu_{u}\left(x_{k}, y_{k}, U_{i}\right)}{\sum_{i} \mu_{U} \cdot\left(x_{k}, y_{k}, U_{i}\right)}
$$

Where $\operatorname{COG}\left(x_{k}, y_{k}\right)$ and $\operatorname{COA}\left(x_{k}, y_{k}\right)$ illustrates the crisp value of defuzzification output, $U_{i} \in U$ is discrete element of an output of the fuzzy set, $\mu_{U} \cdot\left(x_{k}, y_{k}, U_{i}\right)$ is the fuzzy set membership function, and $r$ is the number of fuzzy rules.

Based on foundation of fuzzy logic methodology; fuzzy logic controller has played important rule to design nonlinear controller for nonlinear and uncertain systems [53-66]. However the application area for fuzzy control is really wide, the basic form for all command types of controllers consists of;

- Input fuzzification (binary-to-fuzzy[B/F]conversion)

- Fuzzy rule base (knowledge base)

- Inference engine

- Output defuzzification (fuzzy-to-binary $[\mathrm{F} / \mathrm{B}]$ conversion).

Figure 1 shows the part in fuzzy logic theory.

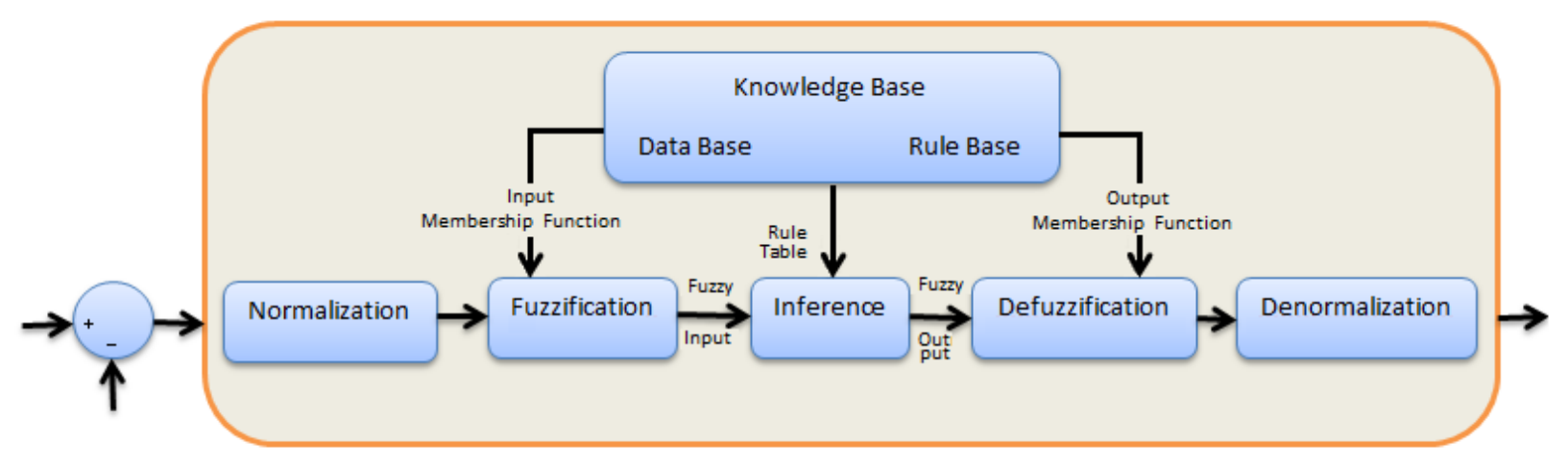

Fig. 1: Block diagram of Fuzzy Logic Control

Feedback linearization Controller: Feedback linearization Controller (FLC) is one of the powerful nonlinear methodology, which is used in nonlinear certain and partly uncertain systems [30-53].This methodology is used in wide range areas such as in control access process, in aerospace applications, in robotic and in electrical motors, to solve some main challenging topics in control such as resistivity to the external disturbance and stability. Even though, this methodology is used in wide range areas but, pure computed torque controller dependence on the system dynamics that is the main challenge. The central idea of feedback linearization controller (FLC) is feedback linearization method. It has assumed that the desired motion trajectory for the manipulator $\boldsymbol{q}_{\boldsymbol{d}}(\boldsymbol{t})$, as determined, by a path planner. Defines the tracking error as [23-37]:

$$
e(t)=q_{d}(t)-q_{a}(t)
$$

Where e(t) is error of the plant, $\boldsymbol{q}_{\boldsymbol{d}}(\boldsymbol{t})$ is desired input variable, that in our system is desired displacement, $\boldsymbol{q}_{\boldsymbol{a}}(\boldsymbol{t})$ is actual displacement. If an alternative linear state-space equation in the form $\dot{\boldsymbol{x}}=\boldsymbol{A} \boldsymbol{x}+\boldsymbol{B} \boldsymbol{U}$ can be defined as

$$
\dot{x}=\left[\begin{array}{ll}
\mathbf{0} & I \\
\mathbf{0} & \mathbf{0}
\end{array}\right] \boldsymbol{x}+\left[\begin{array}{l}
\mathbf{0} \\
\boldsymbol{I}
\end{array}\right] U
$$

With $\boldsymbol{U}=-\boldsymbol{H}^{-\mathbf{1}}(\boldsymbol{q}) \cdot \boldsymbol{N}(\boldsymbol{q}, \dot{\boldsymbol{q}})+\boldsymbol{H}^{-\mathbf{1}}(\boldsymbol{q}) \cdot \boldsymbol{\tau}$ and this is known as the Brunousky canonical form. By equation (8) and (17) the Brunousky canonical form can be written in terms of the state $\boldsymbol{x}=\left[\boldsymbol{e}^{T} \dot{e}^{T}\right]^{T}$ as $[11-34]$ :

$$
\frac{d}{d t}\left[\begin{array}{l}
e \\
\dot{e}
\end{array}\right]=\left[\begin{array}{ll}
0 & I \\
0 & 0
\end{array}\right] \cdot\left[\begin{array}{l}
e \\
\dot{e}
\end{array}\right]+\left[\begin{array}{l}
0 \\
I
\end{array}\right] U
$$

With

$$
U=\ddot{q}_{d}+H^{-1}(q) \cdot\{N(q \cdot \dot{q})-\tau\}
$$

Then compute the required arm torques using inverse of equation (20), is;

$$
\tau=H(q)\left(\ddot{q_{d}}-U\right)+N(\dot{q}, q)
$$


This is a nonlinear feedback control law that guarantees tracking of desired trajectory. Selecting proportional-plus-derivative (PD) feedback for $\mathrm{U}(\mathrm{t})$ results in the PD-computed torque controller [8-10];

$$
\tau=H(q)\left(\ddot{q}_{d}+K_{v} \dot{e}+K_{p} e\right)+N(q, \dot{q})
$$

Where $\boldsymbol{N}(\boldsymbol{q}, \dot{\boldsymbol{q}})$ is nonlinear term of system dynamic;

$$
N(\boldsymbol{q}, \dot{\boldsymbol{q}})=B_{(q)}[\dot{q} \dot{q}]+C_{(q)}[\dot{q}]^{2}
$$

According to the linear system theory, convergence of the tracking error to zero is guaranteed [6]. Where $\boldsymbol{K}_{\boldsymbol{p}}$ and $\boldsymbol{K}_{\boldsymbol{v}}$ are the controller gains.

Figure 2 shows the block diagram of nonlinear computed torque controller with application to spherical motor.

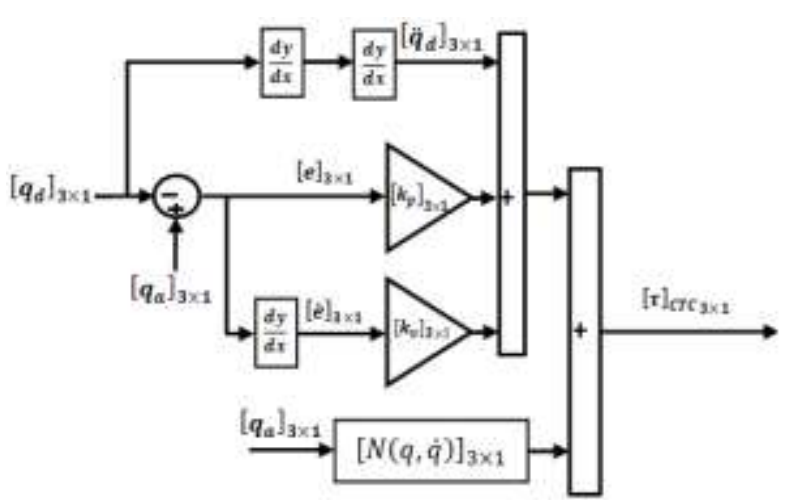

Fig. 2: Block diagram of computed torque Controller

\section{Methodology}

Fuzzy logic controller is one of the most vital influential intelligent nonlinear robust controllers which are used in several applications such as in spherical motor, robotics, process control, aerospace, and power electronics. The main reasons to choose this controller in wide range of application are; free of dynamic modeling of system, suitable control performance and solve some main challenging topics in control. Conversely pure fuzzy logic controller is used in wide range areas it has following mainly important disadvantages i.e. reliability and design a accurate dynamic formulate related to rule table. To reduce or remove above problems feedback linearization controller is applied to fuzzy logic controller to increase the reliability and robustness. The main goal in feedback linearization like controller is applied feedback linearization controller to pure fuzzy logic controller in order to resolve most important problems in pure fuzzy logic controller. This research focuses on resolve the reliability in fuzzy logic theory according to the model-base feedback linearization controller. When feedback linearization methodology applied to pure fuzzy logic controller, fuzzy logic method can compensate the nonlinearity of dynamic equivalent parameters in feedback linearization methodology. This technique was employed to obtain the desired control behavior with a number of information about dynamic model of system and a fuzzy was applied to reinforce system performance.

Based on Fig 2 feedback linearization controller has two main important parts: linear and nonlinear part.

$$
\begin{aligned}
& U_{F L C}=H(q)\left(\ddot{q}_{d}+K_{v} \dot{e}+K_{p} e\right)+ \\
& B_{(q)}[\dot{q} \dot{q}]+C_{(q)}[\dot{q}]^{2}
\end{aligned}
$$

The serial feedback linearization like controller's output is written;

$$
\hat{\boldsymbol{\tau}}_{\text {tot }}=U_{\text {fuzzy }} \times U_{F L C}
$$

Based on fuzzy logic methodology

$$
f(x)=U_{f u z z y}=\sum_{l=1}^{M} \theta^{T} \zeta(x)
$$

where $\boldsymbol{\theta}^{\boldsymbol{T}}$ is adjustable parameter (gain updating factor) and $\boldsymbol{\zeta}(\boldsymbol{x})$ is defined by;

$$
\zeta(x)=\frac{\sum_{i} \mu\left(x_{i}\right) x_{i}}{\sum_{i} \mu\left(x_{i}\right)}
$$

Feedback linearization like controller is a fuzzy controller that increase the robust and stability by serial feedback linearization controller.

$$
\begin{aligned}
\tau_{t o t}=\sum_{l=1}^{M} \theta^{T} \zeta(x) & \\
\times & {\left[H(q)\left(\ddot{q}_{d}+K_{v} \dot{e}+K_{p} e\right)\right.} \\
& \left.+B_{(q)}[\dot{q} \dot{q}]+C_{(q)}[\dot{q}]^{2}\right]
\end{aligned}
$$

This method is suitable for real-time control applications when powerful processors, which can execute complex algorithms rapidly, are not accessible. In this research fuzzy logic controller is used as a main controller which it has one input related to the feedback linearization controller's output $\left(\boldsymbol{U}_{\boldsymbol{C T C}}\right)$ and one output $\left(\tau_{\text {tot }}\right)$. Input and output are normalized between [-6 to 6]. Input has seven linguistic variables and all of linguistic variables have triangular membership function. The MAMDANI fuzzy inference system is used in this research. Therefore to model the dynamic of fuzzy controller 7 rule bases are design based on MAMDANI fuzzy inference system. The center of gravity (COG) is used as a defuzzification. Four steps are used to design fuzzy logic controller, namely, fuzzification, fuzzy rule base and rule evaluation, aggregation of the rule output (fuzzy inference system) and defuzzification.

Fuzzification: the first step in fuzzification is determine inputs and outputs which, it has one input $\left(\boldsymbol{U}_{\boldsymbol{C T C}}\right)$ and one output $\left(\boldsymbol{\tau}_{\text {tot }}\right)$. The input of this system is the output of the feedback linearization controller. The 
second step is chosen an appropriate membership function for input and output which, to simplicity in implementation because it is a linear function with regard to acceptable performance triangular membership function is selected in this research. The third step is chosen the correct labels for each fuzzy set which, in this research namely as linguistic variable. Based on experience knowledge the linguistic variables for the torque output of feedback linearization controller $\left(\boldsymbol{U}_{\boldsymbol{C T C}}\right)$ are; Negative Big (NB), Negative Medium (NM), Negative Small (NS), Zero (Z), Positive Small (PS), Positive Medium (PM), Positive Big (PB), and experience knowledge it is quantized into thirteen levels represented by: $-6,-5,-0.4,-3,-2,-1,0,1,2,3,4,5,6$ The linguistic variables to find the output $\left(\boldsymbol{\tau}_{\text {tot }}\right)$ are; Large Left (LL), Medium Left (ML), Small Left (SL), Zero (Z), Small Right (SR), Medium Right (MR), Large Right (LR) and it is quantized in to thirteen levels represented by: $-6,-5,-0.4,-3,-2,-1,0,1,2,3,4,5,6$.

Fuzzy rule base and rule evaluation: the first step in rule base and evaluation is to provide a least structured method to derive the fuzzy rule base which, expert experience and control engineering knowledge is used because this method is the least structure of the other one and the researcher derivation the fuzzy rule base from the knowledge of system operate and/or the classical controller. Design the rule base of fuzzy inference system can play important role to design the best performance of parallel fuzzy plus computed torque controller, that to calculate the fuzzy rule base the researcher is used to heuristic method which, it is based on the behavior of the control of robot manipulator. The complete rule base for this controller is shown in Table 1 . Rule evaluation focuses on operation in the antecedent of the fuzzy rules in fuzzy sliding mode controller. This part is used $A N D / O R$ fuzzy operation in antecedent part which $A N D$ operation is used.

Table 1: Rule table of fuzzy logic controller

\begin{tabular}{|l|l|l|l|l|l|l|l|}
\hline$U_{C T C}$ & NB & NM & NS & Z & PS & PM & PB \\
\hline$\tau_{T O T}$ & LR & MR & SR & Z & SL & ML & LL \\
\hline
\end{tabular}

Aggregation of the rule output (Fuzzy inference): based on fuzzy methodology, Max-Min aggregation is used in this work.

Defuzzification: The last step to design fuzzy inference in our parallel fuzzy compensator plus computed torque controller is defuzzification. This part is used to transform fuzzy set to crisp set, therefore the input for defuzzification is the aggregate output and the output of it is a crisp number. Based on fuzzy methodology Center of gravity method $(C O G)$ is used in this research.

Figure 3 shows the MAMDANI feedback linearization like controller with application to spherical motor.

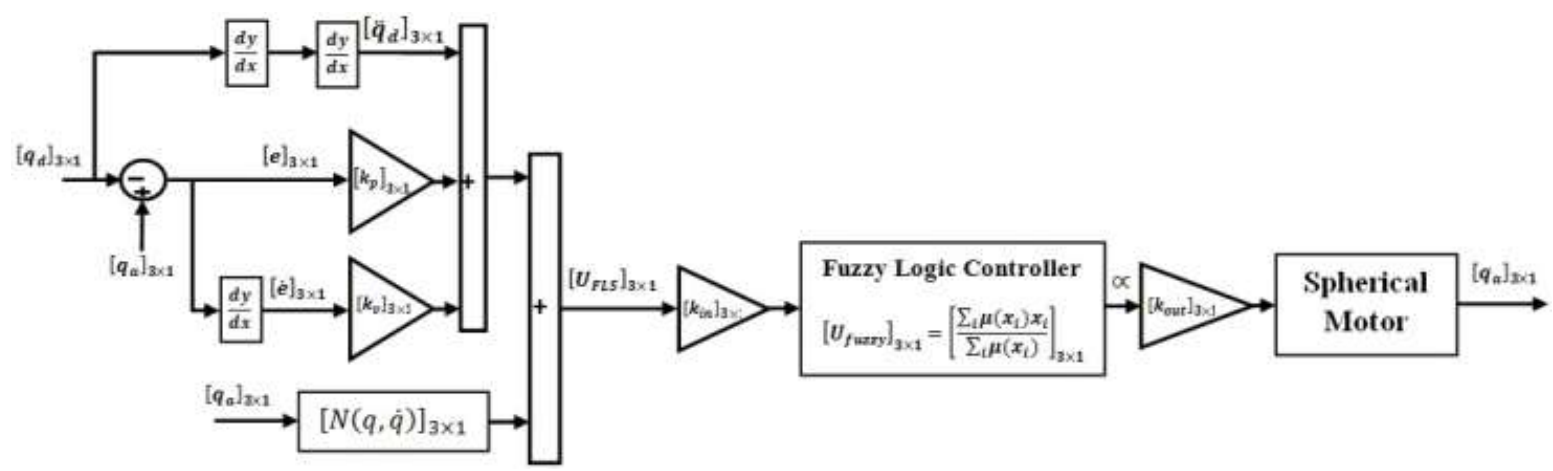

Fig. 3: Block diagram of feedback linearization like controller

Where a matrix of proportional coefficient is $\left[K_{p}\right]_{3 \times 1},\left[K_{v}\right]_{3 \times 1}$ is a matrix of derivative coefficient, , $\left[K_{\text {in }}\right]_{3 \times 1},\left[K_{\text {out }}\right]_{3 \times 1}$ are matrix of gain updating factor.

\section{Result and Discussion}

Feedback linearization like controller and feedback linearization controller are implemented in MATLAB/SIMULINK environment. Tracking performance and disturbance rejection are compared for the following trajectory.
Tracking performances: From the simulation for first, second and third joints (spherical joints) without any disturbance, it was seen that proposed controller and feedback linearization method have good trajectory performance, because these controllers are adjusted and worked on certain environment. Figure 4 shows the tracking performance in certain conditions. 

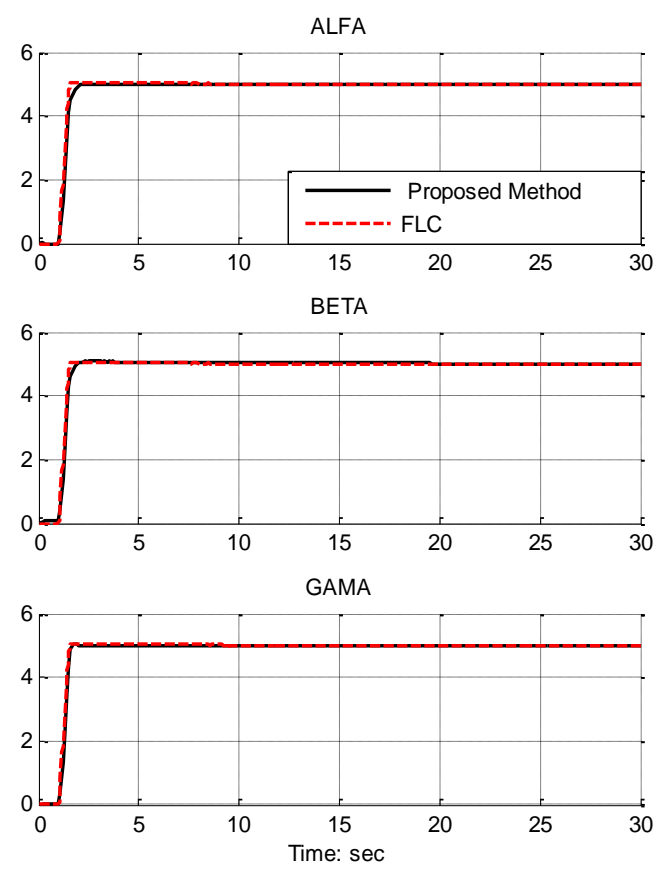

Fig. 4: Proposed Methodology and feedback linearization controller applied to spherical motor without disturbance

According to Figure 4, these types of controllers are model-base therefore they have acceptable performance in certain condition.
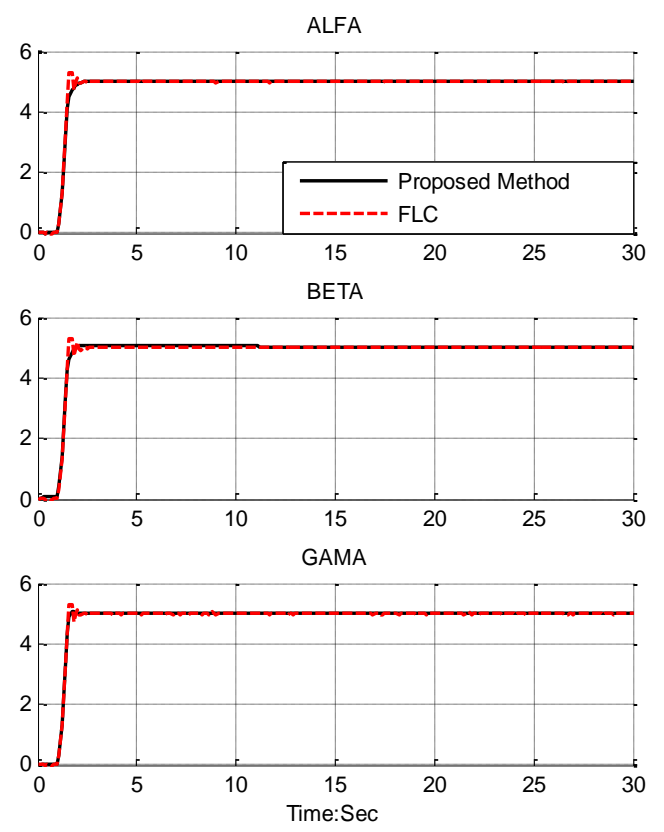

Fig. 5: Proposed method Vs. Feedback linearization control in presence of uncertainty and external disturbance: applied to spherical motor

Disturbance rejection: Figure 5 shows the power disturbance elimination in pure feedback linearization controller and proposed method. The main objectives in these controllers are disturbance rejection, trajectory following and reduce the error. A band limited white noise with predefined of $40 \%$ the power of input signal is applied to controllers. It found fairly fluctuations in FLC trajectory responses. Among following graphs relating to trajectory following with external disturbance, FLC has fairly fluctuations.

According to above graph, feedback linearization methodology has overshoot and moderate oscillation but proposed method can reject these two challenges.

\section{Conclusion}

According to the dynamic formulation of spherical motor; this system is highly nonlinear and uncertain dynamic parameters. Control of this system based on pure soft computing methodology and pure classical methodology is very complicated. The main contribution in this paper is design feedback linearization controller based on the dynamic formulation of system and applied this controller to the main controller (fuzzy logic controller) in serially. This method is test by MATLAB/SIMULINK according to two important test; trajectory following and disturbance rejection. Proposed method can reject the overshoot and oscillation in presence of uncertainty and external disturbance but pure classical nonlinear controller has problem.

\section{Acknowledgment}

The authors would like to thank the anonymous reviewers for their careful reading of this paper and for their helpful comments. This work was supported by the SSP Institute of Advance Science and Technology Program of Iran under grant no. 2013-Persian Gulf-2A.

\section{References}

[1] Vachtsevanos, G. I., Davey, K. and Lee, K. M., "Development of a Novel Intelligent Robotic Manipulator," IEEE Control System Magazine, 1987, pp.9-15.

[2] Davey, K., Vachtsevanos, G. I., and Powers, R., "An analysis of Fields and Torques in Spherical Induction Motors," lEE Transactions on Magnetics, Vol. MAG-23, 1987, pp. 273-282.

[3] Foggia, A., Oliver, E., Chappuis, F., "New Three Degrees of Freedom Electromagnetic Actuator," Conference Record -1AS Annual Meeting, Vol. 35, New York, 1988.

[4] Lee, K. M., Vachtsevanos, G. and Kwan, C-K., "Development of a Spherical Wrist Stepper Motor," Proceedings of the 1988 IEEE Intemational Conference on Robotics and Automation, Philadelphia, PA. April 26-29.

[5] Lee, K. M., Pei. I., "Kinematic Analysis of a Three Degree-of-Freedom Spherical Wrist Actuator," The 
Fifth International Conference on Advanced Robotics, Italy, 1991.

[6] Wang, I., Jewel, G., Howe, D., "Modeling of a Novel Spherical Pennanent Magnet Actuator," Proceedings of IEEE International Conference on Robotics and Automation, Albuquerque, New Mexico, pp 1190-1195, 1997.

[7] Wang, I., Jewel, G., Howe, D., "Analysis, Design and Control of a Novel Spherical Pennanent Magnet Actuator," 1EE Proceedings on Electrical Power Applications., vol. 154, no. 1, 1998.

[8] Chirikjian, G. S., and Stein, D., "Kinematic Design and Commutation of a Spherical Stepper Motor," IEEEIASME Transactions on Mechatronics, vol. 4, n 4, Piscataway, New Jersey, pp. 342-353, Dec. 1999.

[9] Kahlen, K., and De Doncker, R. W., "CW'l'ent Regulators for Multi-phase Pennanent Magnet Spherical Machines." Industry Applications Conference Record of the 2000 IEEE, vol. 3, 2000, pp. 2011-2016.

[10] Lee, K. M., Pei, I., and Gilboa, U., "On the Development of a Spherical Wrist Actuator," Proceedings of the 16th NSF Conference on Manufacturing Systems Research, Tempe AZ, January 8-12, 1990.

[11] Yang, C., Back, Y. S., "Design and Control of the 3-dcgn:es of freedom actuator by Controlling the Electromagnetic Force," IEEE Transactions on Magnetics, May, 1999, pp. 3607-3609.

[12] Samira Soltani \& Farzin Piltan, "Design Artificial Nonlinear Controller Based on Computed Torque like Controller with Tunable Gain”, World Applied Science Journal (WASJ), 14 (9): 1306-1312, 2011.

[13] Farzin Piltan, Mohammadali Dialame, Abbas Zare \& Ali Badri,"Design Novel Lookup Table Changed Auto Tuning FSMC:Applied to Robot Manipulator", International Journal of Engineering, $6(1): 25-41,2012$

[14] Farzin Piltan, Mohammad Keshavarz, Ali Badri \& Arash Zargari,"Design Novel Nonlinear Controller Applied to RobotManipulator: Design New Feedback Linearization Fuzzy Controller with Minimum Rule Base Tuning Method", International Journal of Robotics and Automation,3 (1):1-12, 2012

[15] Farzin Piltan, Iman Nazari, Sobhan Siamak, Payman Ferdosali,"Methodology of FPGA-Based Mathematical error-Based Tuning Sliding Mode Controller", International Journal of Control and Automation, 5(1), 89-118, 2012

[16] Farzin Piltan, Bamdad Boroomand, Arman Jahed \& Hossein Rezaie, "Methodology of Mathematical Error-Based Tuning Sliding Mode Controller",
International Journal of Engineering, 6 (2):96-117, 2012

[17] Farzin Piltan, Sara Emamzadeh, Zahra Hivand, Fatemeh Shahriyari \& Mina Mirazaei, "PUMA-560 Robot Manipulator Position Sliding Mode Control Methods Using MATLAB/SIMULINK and Their Integration into Graduate/Undergraduate Nonlinear Control, Robotics and MATLAB Courses", International Journal of Robotics and Automation, 3(3):106-150, 2012

[18] Farzin Piltan, Ali Hosainpour, Ebrahim Mazlomian, Mohammad Shamsodini, Mohammad H. Yarmahmoudi, "Online Tuning Chattering Free Sliding Mode Fuzzy Control Design: Lyapunov Approach", International Journal of Robotics and Automation, 3(3):77-105, 2012

[19] Farzin Piltan, Mina Mirzaei, Forouzan Shahriari, Iman Nazari, Sara Emamzadeh, "Design Baseline Computed Torque Controller", International Journal of Engineering, 6(3): 129-141, 2012

[20] Farzin Piltan, Mohammad H. Yarmahmoudi, Mohammad Shamsodini, Ebrahim Mazlomian, Ali Hosainpour, "PUMA-560 Robot Manipulator Position Computed Torque Control Methods Using MATLAB/SIMULINK and Their Integration into Graduate Nonlinear Control and MATLAB Courses", International Journal of Robotics and Automation, 3(3): 167-191, 2012

[21] Farzin Piltan, Hossein Rezaie, Bamdad Boroomand, Arman Jahed, "Design Robust Backstepping online Tuning Feedback Linearization Control Applied to IC Engine", International Journal of Advance Science and Technology, 11:40-22, 2012

[22] Farzin Piltan, Mohammad R. Rashidian, Mohammad Shamsodini and Sadeq Allahdadi, Effect of Rule Base on the Fuzzy-Based Tuning Fuzzy Sliding Mode Controller: Applied to $2^{\text {nd }}$ Order Nonlinear System", International Journal of Advanced Science and Technology, 46:39-70, 2012

[23] Farzin Piltan, Arman Jahed, Hossein Rezaie and Bamdad Boroomand, "Methodology of Robust Linear On-line High Speed Tuning for Stable Sliding Mode Controller: Applied to Nonlinear System", International Journal of Control and Automation, 5(3): 217-236, 2012

[24] Farzin Piltan, Bamdad Boroomand, Arman Jahed and Hossein Rezaie, "Performance-Based Adaptive Gradient Descent Optimal Coefficient Fuzzy Sliding Mode Methodology", International Journal of Intelligent Systems and Applications, , vol.4, no.11, pp.40-52, 2012.

[25] Farzin Piltan, Mehdi Akbari, Mojdeh Piran , Mansour Bazregar, "Design Model Free Switching Gain Scheduling Baseline Controller with Application to Automotive Engine", International Journal of Information Technology and Computer 
Science, vol.5, no.1, pp.65-73, 2013.DOI: 10.5815/ijitcs.2013.01.07.

[26] Farzin Piltan, Mojdeh Piran , Mansour Bazregar, Mehdi Akbari, "Design High Impact Fuzzy Baseline Variable Structure Methodology to Artificial Adjust Fuel Ratio", International Journal of Intelligent Systems and Applications, vol.5, no.2, pp.59-70, 2013.DOI: 10.5815/ijisa.2013.02.0.

[27] Farzin Piltan, M. Bazregar, M. kamgari, M. Akbari and M. Piran, "Adjust the Fuel Ratio by High Impact Chattering Free Sliding Methodology with Application to Automotive Engine", International Journal of Hybrid Information Technology, 6(1), 2013.

[28] Farzin Piltan, S. Zare , F. ShahryarZadeh, M. Mansoorzadeh, M. kamgari, "Supervised Optimization of Fuel Ratio in IC Engine Based on Design Baseline Computed Fuel Methodology", International Journal of Information Technology and Computer Science, vol.5, no.4, pp.76-84, 2013.DOI: 10.5815/ijitcs.2013.04.09.

[29] Farzin Piltan, M. Mansoorzadeh, S. Zare, F.Shahryarzadeh, M. Akbari, "Artificial Tune of Fuel Ratio: Design a Novel SISO Fuzzy Backstepping Adaptive Variable Structure Control”, International Journal of Electrical and Computer Engineering, 3(2), 2013.

[30] M. Bazregar, Farzin Piltan, A. Nabaee and M.M. Ebrahimi, "Parallel Soft Computing Control Optimization Algorithm for Uncertainty Dynamic Systems", International Journal of Advanced Science and Technology, 51, 2013.

[31] Farzin Piltan, M.H. Yarmahmoudi, M. Mirzaei, S. Emamzadeh, Z. Hivand, "Design Novel Fuzzy Robust Feedback Linearization Control with Application to Robot Manipulator", International Journal of Intelligent Systems and Applications , vol.5, no.5, pp.1-10, 2013.DOI: 10.5815/ijisa.2013.05.01

[32] Sh. Tayebi Haghighi, S. Soltani, Farzin Piltan, M. kamgari, S. Zare, "Evaluation Performance of IC Engine: Linear Tunable Gain Computed Torque Controller Vs. Sliding Mode Controller", International Journal of Intelligent Systems and Applications, vol.5, no.6, pp.78-88, 2013.DOI: 10.5815/ijisa.2013.06.10.

[33] Amin Jalali, Farzin Piltan, M. Keshtgar, M. Jalali, "Colonial Competitive Optimization Sliding Mode Controller with Application to Robot Manipulator", International Journal of Intelligent Systems and Applications, vol.5, no.7, pp.50-56, 2013. DOI: 10.5815/ijisa.2013.07.07.

[34] Salehi, Farzin Piltan, M. Mousavi, A. Khajeh, M. R. Rashidian, "Intelligent Robust Feed-forward Fuzzy Feedback Linearization Estimation of PID Control with Application to Continuum Robot",
International Journal of Information Engineering and Electronic Business, vol.5, no.1, pp.1-16, 2013. DOI: 10.5815/ijieeb.2013.01.01.

[35] Farzin Piltan, M.J. Rafaati, F. Khazaeni, A. Hosainpour, S. Soltani, "A Design High Impact Lyapunov Fuzzy PD-Plus-Gravity Controller with Application to Rigid Manipulator", International Journal of Information Engineering and Electronic Business, vol.5, no.1, pp.17-25, 2013. DOI: 10.5815/ijieeb.2013.01.02.

[36] Amin Jalali, Farzin Piltan, A. Gavahian, M. Jalali, M. Adibi, "Model-Free Adaptive Fuzzy Sliding Mode Controller Optimized by Particle Swarm for Robot manipulator", International Journal of Information Engineering and Electronic Business, vol.5, no.1, pp.68-78, 2013. DOI: 10.5815/ijieeb.2013.01.08.

[37] Farzin Piltan, F. ShahryarZadeh ,M. Mansoorzadeh, M. kamgari, S. Zare, "Robust Fuzzy PD Method with Parallel Computed Fuel Ratio Estimation Applied to Automotive Engine", International Journal of Intelligent Systems and Applications, vol.5, no.8, pp.83-92, 2013. DOI: 10.5815/ijisa.2013.08.10.

[38] Farzin Piltan, A. Nabaee, M.M. Ebrahimi, M. Bazregar, "Design Robust Fuzzy Sliding Mode Control Technique for Robot Manipulator Systems with Modeling Uncertainties", International Journal of Information Technology and Computer Science, vol.5, no.8, pp.123-135, 2013. DOI: 10.5815/ijitcs.2013.08.12.

[39] Farzin Piltan, M. Mansoorzadeh, M. Akbari, S. Zare, F. ShahryarZadeh "Management of Environmental Pollution by Intelligent Control of Fuel in an Internal Combustion Engine" Global Journal of Biodiversity Science And Management, 3(1), 2013.

[40] M. M. Ebrahimit Farzin Piltan, M. Bazregar and A.R. Nabaee, "Intelligent Robust Fuzzy-Parallel Optimization Control of a Continuum Robot Manipulator", International Journal of Control and Automation, 6(3), 2013.

[41] O.R. Sadrnia, Farzin Piltan, M. Jafari, M. Eram and M. Shamsodini, "Design PID Estimator Fuzzy plus Backstepping to Control of Uncertain Continuum Robot", International Journal of Hybrid Information Technology, 6(4), 2013.

[42] AminJalali, Farzin Piltan, H. Hashemzadeh, A. Hasiri, M.R Hashemzadeh, "Design Novel Soft Computing Backstepping Controller with Application to Nonlinear Dynamic Uncertain System", International Journal of Intelligent Systems and Applications, vol.5, no.10, pp.93-105, 2013. DOI: $10.5815 /$ ijisa.2013.10.12.

[43] M. Moosavi, M. Eram, A. Khajeh, O. Mahmoudi and Farzin Piltan, "Design New Artificial 
Intelligence Base Modified PID Hybrid Controller for Highly Nonlinear System", International Journal of Advanced Science and Technology, 57, 2013.

[44] S. Zahmatkesh, Farzin Piltan, K. Heidari, M. Shamsodini, S. Heidari, "Artificial Error Tuning Based on Design a Novel SISO Fuzzy Backstepping Adaptive Variable Structure Control" International Journal of Intelligent Systems and Applications, vol.5, no.11, pp.34-46, 2013. DOI: 10.5815/ijisa.2013.11.04.

[45] S. Heidari, Farzin Piltan, M. Shamsodini, K. Heidari and S. Zahmatkesh, "Design New Nonlinear Controller with Parallel Fuzzy Inference System Compensator to Control of Continuum Robot Manipulator",International Journal of Control and Automation, 6(4), 2013.

[46] FarzinPiltan, M. Kamgari, S. Zare, F. ShahryarZadeh, M. Mansoorzadeh, "Design Novel Model Reference Artificial Intelligence Based Methodology to Optimized Fuel Ratio in IC Engine", International Journal of Information Engineering and Electronic Business, vol.5, no.2, pp.44-51, 2013. DOI: 10.5815/ijieeb.2013.02.07.

[47] Farzin Piltan, Mehdi Eram, Mohammad Taghavi, Omid Reza Sadrnia, Mahdi Jafari,"Nonlinear Fuzzy Model-base Technique to Compensate Highly Nonlinear Continuum Robot Manipulator", IJISA, vol.5, no.12, pp.135-148, 2013. DOI: 10.5815/ijisa.2013.12.12

[48] Amin Jalali, Farzin Piltan, Mohammadreza Hashemzadeh, Fatemeh BibakVaravi, Hossein Hashemzadeh,"Design Parallel Linear PD Compensation by Fuzzy Sliding Compensator for Continuum Robot", IJITCS, vol.5, no.12, pp.97112, 2013. DOI: 10.5815/ijitcs.2013.12.12

[49] Farzin Piltan, A. Hosainpour, S. Emamzadeh, I. Nazari, M. Mirzaie, "Design Sliding Mode Controller of with Parallel Fuzzy Inference System Compensator to Control of Robot Manipulator", International Journal of Robotics and Automation, Vol. 2, No. 4, December 2013, pp. 149 162.

[50] Farzin Piltan, Mahdi Jafari, Mehdi Eram, Omid Mahmoudi, Omid Reza Sadrnia, "Design Artificial Intelligence-Based Switching PD plus Gravity for Highly Nonlinear Second Order System", International Journal of Engineering and Manufacturing, vol.3, no.1, pp.38-57, 2013.DOI: 10.5815/ijem.2013.01.04

[51] Farzin Piltan, Sara Emamzadeh, Sara Heidari, Samaneh Zahmatkesh, Kamran Heidari, "Design Artificial Intelligent Parallel Feedback Linearization of PID Control with Application to Continuum Robot", International Journal of Engineering and Manufacturing, vol.3, no.2, pp.5172, 2013.DOI: 10.5815/ijem.2013.02.04
[52] Mohammad Mahdi Ebrahimi, Farzin Piltan, Mansour Bazregar, AliReza Nabaee,"Artificial Chattering Free on-line Modified Sliding Mode Algorithm: Applied in Continuum Robot Manipulator", International Journal of Information Engineering and Electronic Business, vol.5, no.5, pp.57-69, 2013. DOI: 10.5815/ijieeb.2013.05.08

[53] Arman Jahed, Farzin Piltan, Hossein Rezaie, Bamdad Boroomand, "Design Computed Torque Controller with Parallel Fuzzy Inference System Compensator to Control of Robot Manipulator", International Journal of Information Engineering and Electronic Business, vol.5, no.3, pp.66-77, 2013. DOI: 10.5815/ijieeb.2013.03.08

[54] Mohammad Shamsodini, Farzin Piltan, Mahdi Jafari, Omid reza Sadrnia, Omid Mahmoudi,"Design Modified Fuzzy Hybrid Technique: Tuning By GDO", IJMECS, vol.5, no.8, pp.58-72, 2013.DOI: 10.5815/ijmecs.2013.08.07

[55] Mahdi Mirshekaran, Farzin Piltan,Zahra Esmaeili, Tannaz Khajeaian, Meysam Kazeminasab,"Design Sliding Mode Modified Fuzzy Linear Controller with Application to Flexible Robot Manipulator", IJMECS, vol.5, no.10, pp.53-63, 2013.DOI: 10.5815/ijmecs.2013.10.07

[56] Meysam Kazeminasab, Farzin Piltan, Zahra Esmaeili, Mahdi Mirshekaran, Alireza Salehi ,"Design Parallel Fuzzy Partly Inverse Dynamic Method plus Gravity Control for Highly Nonlinear Continuum Robot", IJISA, vol.6, no.1, pp.112-123, 2014. DOI: 10.5815/ijisa.2014.01.12.

[57] Mansour Bazregar, Farzin Piltan, Mehdi Akbari, Mojdeh Piran,"Management of Automotive Engine Based on Stable Fuzzy Technique with Parallel Sliding Mode Optimization", IJITCS, vol.6, no.1, pp.101-107, 2014. DOI: 10.5815/ijitcs.2014.01.12.

\section{Authors' Profiles}

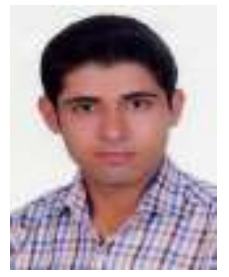

Ali Barzegar is currently working as a co researcher in Control and Robotic Lab at the institute of advance science and technology, IRAN SSP research and development Center. His current research interests are in the area of nonlinear control, artificial control system and robotics, and spherical motor.

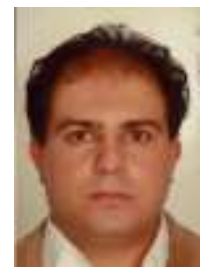

Farzin Piltan was born on 1975, Shiraz, Iran. In 2004 he is jointed Institute of Advance Science and Technology, Research and Development Center, IRAN SSP. Now he is a dean of Intelligent Control and Robotics Lab. In addition to 7 textbooks, Farzin Piltan is the main author of more than 100 scientific papers in 
refereed journals. He is editorial review board member for 'international journal of control and automation (IJCA), Australia, ISSN: 2005-4297; 'International Journal of Intelligent System and Applications (IJISA)', Hong Kong, ISSN: 2074-9058; 'IAES international journal of robotics and automation, Malaysia, ISSN: 2089-4856; 'International Journal of Reconfigurable and Embedded Systems', Malaysia, ISSN: 2089-4864. His current research interests are nonlinear control, artificial control system and applied to FPGA, robotics and artificial nonlinear control and IC engine modeling and control.

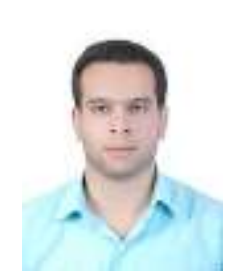

Mahmood Vosoogh is currently working as a co researcher in Control and Robotic Lab at the institute of advance science and technology, IRAN SSP research and development Center. His current research interests are in the area of nonlinear control, artificial control system and robotics, and spherical motor.

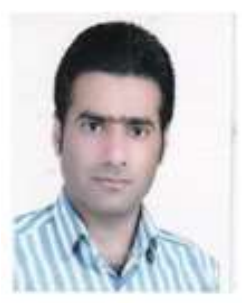

Abdol Majid Mirshekaran is currently working as a co researcher in Control and Robotic Lab at the institute of advance science and technology, IRAN SSP research and development Center. He is a Master in field of Electrical Engineering from Islamic Azad University, IRAN. His current research interests are in the area of nonlinear control, artificial control system and robotics, and spherical motor.

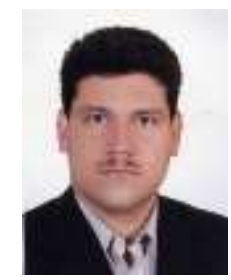

AliReza Siahbazi is currently working as a co researcher in Control and Robotic Lab at the institute of advance science and technology, IRAN SSP research and development Center. $\mathrm{He}$ is a Master in field of Computer Engineering from Shiraz University, Shiraz, IRAN. His current research interests are in the area of nonlinear control, artificial control system and robotics, and spherical motor.

How to cite this paper: Ali Barzegar, Farzin Piltan, Mahmood Vosoogh, Abdol Majid Mirshekaran, Alireza Siahbazi,"Design Serial Intelligent Modified Feedback Linearization like Controller with Application to Spherical Motor", International Journal of Information Technology and Computer Science(IJITCS), vol.6, no.5, pp.72-83, 2014. DOI: 10.5815/ijitcs.2014.05.10 